Article

\title{
Calibration of Melt, Shear Modulus, and Flow Stress Models for Cerium Subjected to Intensive Dynamic Loading
}

\author{
Marvin A. Zocher * (D), JeeYeon N. Plohr and Leonid Burakovsky \\ Los Alamos National Laboratory, Los Alamos, NM 87545, USA; jplohr@lanl.gov (J.N.P.); burakov@lanl.gov (L.B.) \\ * Correspondence: zocher@lanl.gov
}

Received: 15 August 2020; Accepted: 6 October 2020; Published: 16 October 2020

check for updates

\begin{abstract}
Calibration parameters are developed for melt, shear modulus, and flow stress models for cerium subjected to dynamic loading. Parametric calibration is developed for the Lindemann melt law and for the shear modulus and flow stress models of Steinberg, Cochran, and Guinan.
\end{abstract}

Keywords: cerium; melt; shear modulus; flow stress

\section{Introduction}

The continuum-level thermo-mechanical problem of predicting the behavior of ductile metallic components subjected to intensive dynamic loading and undergoing plastic flow is of central importance in a number of applications. Consequently, this is a field of study that has received significant attention over at least the last 170 years. However, even with such sustained attention, the problem remains one that is not fully resolved. One reason for this is that closed form solutions of the initial boundary value problem (IBVP) are almost always untenable, and numerical approaches are inherently limited by approximations that the modeler is forced make.

Numerical solution of the IBVP requires the simultaneous solution of a coupled set of governing field equations (typically: conservation of mass, conservation of linear momentum, and conservation of energy), along with an appropriate set of initial and boundary conditions. Note that within the context of plasticy, resultant from intensive dynamic loading, the field equations are typically formulated in rate form. That being the case, they must be solved in incremental fashion as time goes on. Together, the governing field equations form a hyperbolic set, for which a numerical solution may be attempted using Lagrangian, Eulerian, or Augmented Lagrangian-Eulerian (ALE) approaches employing either explicit or implicit numerical time integration schemes depending on the specifics of the IBVP and preferences of the modeler. Numerical approaches include the finite element method and the finite volume method, among others.

If all one has to work with are the governing field equations plus initial and boundary conditions, the IBVP is inherently underconstrained and as such is ill-posed. This is because there are more unknown variables than equations to constrain them. It is through certain constitutive equations that the IBVP attains closure and is well-posed, and the solution of the same becomes tenable. There are a number of constitutive relations that may be critical in this regard, but the focus of this work is on only one-namely that related to the deviatoric Cauchy stress, and it is within the context of classical plasticity that it is herein addressed.

By way of review, classical plasticity consists of the following fundamental parts: (1) a stress-strain relationship (the constitutive equations that are required for closure of the IBVP), and (2) several component parts that feed into the constitutive equations. Those component parts include a yield criterion, a flow rule, and a hardening rule. Note that the hardening rule includes, or should 
include, mechanisms for softening as well as hardening, so it should perhaps be referred to as a hardening/softening rule, but we shall, out of adherence to convention, refer to it herein as a hardening rule with the understanding that mechanisms for softening are included. The stress-strain equations can be formulated in several different ways. One possibility is an algebraic relationship between the Cauchy stress and Eulerian strain:

$$
\sigma_{i j}=C_{i j k l}\left(\epsilon_{k l}-\epsilon_{k l}^{p}\right)
$$

where $\sigma_{i j}$ is the Cauchy stress, $C_{i j k l}$ is a fourth order tensor of material properties, $\epsilon_{k l}$ is the Eulerian strain, and the superscript $p$ is used to denote the plastic part of the strain tensor. Another possibility is to separate the Cauchy tensor into deviatoric and spherical parts and to focus our plasticity-related attention exclusively on the deviator since it is within the deviator that plastic flow characteristics normally manifest. In this case, the spherical part of the Cauchy tensor is determined separately-for example, through something like an equation of state (constitutive relationship for the spherical part of the Cauchy tensor). Taking this approach, as is most common in the field of plasticity resultant from intensive dynamic loading, the constitutive equations are typically formulated, not in terms of the deviator directly, but in terms of a deviatoric stress rate, $\dot{s}_{i j}$, and may be expressed as follows:

$$
\dot{s}_{i j}=C_{i j k l}\left(\eta_{k l}-\eta_{k l}^{p}\right)
$$

where $s_{i j}$ is the deviatoric stress, $C_{i j k l}$ is, as above, a fourth order tensor of material properties, $\eta_{k l}$ denotes the deviatoric part of the rate of deformation tensor (deviatoric natural strain rate) [1], and the superscript $p$ denotes the plastic part of the rate of deformation tensor. Note that, for isotropic systems, $C_{i j k l}$ simplifies to $2 G$, where $G$ is the shear modulus. The deviatoric stress rate is typically cast in terms of a Jaumann stress rate or, alternatively, a Green-Nagdi stress rate [2]. Note that the choice of formulating the constitutive equations in rate form is natural since the governing field equations, as mentioned earlier, are most often expressed in rate form, and must, consequently, be solved in an incremental fashion over time with an update of the the stress-strain relationship occurring on each time step. Whatever form the constitutive equations take, they should be developed in accordance with four fundamental principles: (1) the principle of local action (if appropriate), (2) the principle of equipresence, (3) the principle of determinism (assures uniqueness), and (4) the principle of frame indifference [3]. Please note that, although not shown explicitly in Equations (1) and (2), $\sigma_{i j}$ and $s_{i j}$ are functions of temperature as well as whatever measure of strain is chosen as pertinent.

Let us now briefly address the component parts that feed into the constitutive equations discussed above. The yield criterion is typically formulated in terms of strain, temperature, and possibly some number of internal state variables that evolve over time (i.e., $\left.F=F\left(\epsilon_{k l}, T, \alpha_{k l}^{\mu}\right)\right)$. Options for the precise form of the yield criterion are several. One possibility is to express yield in a stress formulation involving the deviator, for example, as follows:

$$
F=\frac{1}{2} \alpha_{i j k l} s_{i j} s_{i j}-\sigma^{2}=0
$$

where $F$ represents the yield surface, $\alpha_{i j k l}$ is a fourth order tensor of coefficients that define the shape of the yield surface, and $\sigma$ is the flow stress, which incorporates hardening and softening effects. Note that the yield surface, as expressed here, is a function of deviatoric stress only (as opposed to the full Cauchy tensor) and since $s_{k k}=0$, this is a five dimensional space. Note also that von Mises $J_{2}$ plasticity [2] exists as a simplified subset of Equation (3) and is expressed as follows:

$$
F=\frac{1}{2} s_{i j} s_{i j}-\sigma^{2}=0
$$

where $J_{2} \equiv \frac{1}{2} s_{i j} s_{i j}$. 
As was the case with the yield criterion, the flow rule, which is a rule for the evolution of plastic strain and can be thought of as an internal state growth law, can be formulated in terms of strain, temperature, and perhaps some number of internal state variables (i.e., $\left.\dot{\epsilon}_{i j}^{p}=\epsilon_{i j}^{p}\left(\epsilon_{k l}, T, \alpha_{k l}^{\mu}\right)\right)$. However, it is most often given in a stress formulation, as in the Levy-Mises flow rule (which is in fact a special case of the more general Prandtl-Reuss flow rule) and is given as:

$$
d \epsilon_{i j}^{p}=d \lambda s_{i j}
$$

where $\lambda$ is a history-dependent scalar and all other variables are as already defined. An often-used alternative to the Levy-Mises formulation is what is commonly referred to as an associative flow rule (since it is associated with a particular yield criterion), or the normality condition, and is given as:

$$
d \epsilon_{i j}^{p}=d \lambda \frac{\partial F}{\partial \sigma_{i j}}
$$

However, if we assume that the yield surface is a function of the Cauchy deviator only (as suggested in Equations (3) and (4)), then the normality condition is perhaps more appropriately depicted in terms of the deviator as opposed to the full Cauchy tensor:

$$
d \epsilon_{i j}^{p}=d \lambda \frac{\partial F}{\partial s_{i j}}
$$

where all variables are as already defined.

The final component that feeds into the constitutive equations is the hardening rule. It is a rule for the evolution of the yield surface. This evolution is brought about through the effect of certain internal state varibles, such as the drag stress, $\alpha_{2}$, in the case of isotropic hardening, or the back stress, $\alpha_{3_{i j}}$, in the case of kinematic hardening, along with whatever internal state variable growth laws (i.e., $\left.\dot{\alpha}_{i j}^{\eta}=\alpha_{i j}^{\eta}\left(\epsilon_{k l}, T, \alpha_{k l}^{\mu}\right)\right)$ may be appropriate. In a rather generic form, isotropic and kinematic hardening can be expressed, respectively, by what is given in the two equations that follow:

$$
\begin{gathered}
F=J_{2}-\frac{\alpha_{2}^{2}}{3}=0 \\
F=\frac{1}{2}\left(s_{i j}-\alpha_{3_{i j}}^{\prime}\right)\left(s_{i j}-\alpha_{3_{i j}}^{\prime}\right)-\frac{\sigma^{2}}{3}=0
\end{gathered}
$$

where the superscripted / denotes deviatoric part.

This completes our review of classical plasticity. For a more complex treatment of plasticity-for example, a treatment that includes anisotropy and complications related to material frame indifference- the interested reader may find the publication of Zocher et al. [4] to be of benefit. To keep things simple, going forward we shall limit our consideration to isotropic materials undergoing $J_{2}$ plastic flow in accordance with an associative flow rule and isotropic hardening. Thus, the most pertinent framework for what follows is that represented by Equations (2), (4), (7) and (8). Within that framework, the flow stress relates to the drag stress and to $J_{2}$, as follows:

$$
\sigma=\frac{\alpha_{2}}{\sqrt{3}}=\sqrt{J_{2}}
$$

In what follows, we shall focus our attention on the hardening model only (i.e., Equation (10)), or more precisely, on some specific forms that the generic Equation (10) may take. Those specific forms include the models of Johnson and Cook, [5] (which we shall refer to in "shorthand" fashion as the JC model, though the model is not universally referred to in this manner), Steinburg, Cochran, and Guinan, [6] (which we shall refer to in "shorthand" fashion as the SCG model, though the model is not universally referred to in this manner), Follansbee and Kocks [7] (the MTS model), and Preston, 
Tonks and Wallace [8] (the PTW model). Our ultimate objective will be the development of a hardening model for $\gamma$-phase cerium that is in the style of the SCG model.

Cerium has a very complex phase diagram. There are four solid phases at zero pressure and at least three more high pressure phases. Gamma phase, the stable room temperature zero pressure phase, exhibits unusual hydrostatic constitutive behavior, wherein the bulk modulus decreases with increasing pressure. Cerium is unique in that it is the only pure element possessing a solid-solid critical point. One of the more interesting features of cerium is the isomorphic $\gamma-\alpha$ solid-solid phase transition (fcc to fcc), which occurs at about $0.75 \mathrm{GPa}$ (precipitated by localization/delocalization mechanisms involving $4 \mathrm{f}$ electrons). This transition is accompanied by a substantial volume change of almost $15 \%$ and has a profound impact upon the dynamic response of cerium. For example, the very large volume collapse that occurs with this transition has the effect of causing the temperature to increase at a rate much higher under dynamic compression than would occur with no volume change. For scientific interests and experimental applications, efforts are underway at Los Alamos National Laboratory and elsewhere to build multi-phase constitutive models for cerium. The present work represents a first step in the development of a multi-phase capability for predicting the deviatoric constitutive response of cerium employing an SCG-type hardening model. A corresponding first step was taken by Plohr et al. [9], wherein the focus was on a PTW-type hardening model. An important additional factor motivating the present work derives from activity underway by the first author and others in the development of a multi-scale friction model (see [10]). It turns out, for reasons not pertinent to the present discussion, that the friction model being developed is well suited to the use of a hardening model of SCG-type, but less well suited to the use of some other hardening models. Moreover, since the first author and others wish to test the aforementioned friction model against experiments involving cerium, there is very specific motivation for the present work.

In the following section we shall discuss, very briefly, a few specific hardening models that have appeared in the literature in the "recent" past (in the last 40 years or so). These include the JC model, the SCG model, the MTS model, and the PTW model. Following that, we shall discuss a variant of the PTW model recently developed by Plohr, Burakovsky, and Sjue [9], specifically for $\gamma$-phase cerium. We shall develop a characterization of the SCG model that is applicable to cerium. That characterization will include the paramaterization of the melt model of Lindemann [11] along with models of the shear modulus and flow stress in accordance with SCG. We will then conclude with some summary remarks and recommendations for further study.

\section{Hardening Models}

\subsection{JC}

The Johnson-Cook model is a simple algebraic history independent model within which the flow stress (dynamic yield stress) depends upon the current state of the equivalent plastic strain, equivalent plastic strain rate, and temperature: $\sigma=\sigma(\epsilon, \dot{\epsilon}, T)$. The model is given as:

$$
\sigma=\left[\sigma_{0}+B\left(\epsilon^{p}\right)^{n}\right]\left[1+C \ln \left(\dot{\epsilon}^{p}\right)\right]\left[1-\left(\frac{T-T_{r}}{T_{m}-T_{r}}\right)^{m}\right]
$$

where $\epsilon^{p}$ denotes the equivalent plastic strain, $\dot{\epsilon}^{p}$ denotes the equivalent plastic strain rate, $\sigma_{0}$ denotes the initial yield, $B$ is a strain hardening coefficient, $n$ is a strain hardening exponent, $C$ is a strain rate hardening coefficient, $T_{r}$ denotes room temperature, $T_{m}$ denotes melt temperature, and $m$ is a softening exponent. Hence the first bracketed term in Equation (11) represents strain hardening, the second strain rate hardening, and the third thermal softening. This model has seven input parameters: $\sigma_{0}, B$, $n, C, T_{r}, T_{m}$, and $m$. The equivalent plastic strain rate and equivalent plastic strain are determined as follows:

$$
\dot{\epsilon}^{p}=\sqrt{\frac{2}{3} \eta_{i j} \eta_{i j}}
$$




$$
\epsilon^{p}(t+\Delta t)=\epsilon^{p}(t)+\int_{t}^{t+\Delta t} \dot{\epsilon}^{p} d t
$$

\subsection{PTW}

As with the model of Johnson and Cook, the PTW model is a simple algebraic history independent model within which the flow stress depends upon the current state of the equivalent plastic strain, equivalent plastic strain rate, and temperature: $\sigma=\sigma(\epsilon, \dot{\epsilon}, T)$. That being said, the PTW model represents a significant advancement in capability in that it is applicable over a much wider range of strain rate. This expansion in strain rate applicability is accomplished by "pasting together" what is essentially multiple models, each of which is applicable to a particular strain rate regime. For strain rates up to about $10^{4} \mathrm{~s}^{-1}$, the primary mechanism for plastic flow is thermally activated dislocation glide. For strain rates on the order of $10^{9}$ to $10^{12}$, occurring in what is sometimes referred to as the overdriven shock regime, the predominant mechanism for plastic flow is no longer thermally activated dislocation glide, but dislocation drag. At intermediate strain rates (e.g., $10^{5}$ to $10^{9}$ ) phonon friction may also be decisive. Sub-models within PTW are developed for each of these two strain rate regimes. The gap between strain rates of $10^{4}$ and $10^{9}$ is bridged through simple extrapolation. The PTW model incorporates a model for the shear modulus that is potentially temperature and density dependent. It also incorporates an explicit model for the saturation stress (maxima on a stress-strain curve). The model is structured in such a way that many of its intermediate steps (and many of its input parameters) are dimensionless.

The model shall be presented here in building block fashion involving several sub-models that feed into the flow stress. The first building block is the sub-model for the shear modulus, given as:

$$
G=G_{0}(1-\alpha \widehat{T})
$$

where $G$ is the shear modulus, $\widehat{T}$ is a dimensionless temperature variable defined as: $\widehat{T}=T / T_{m}$ (where $T_{m}$ is the melt temperature), $G_{0}$ is the zero temperature shear modulus, and $\alpha$ is an input parameter. The second building block is $\dot{\xi}$, defined as:

$$
\dot{\xi}=\frac{1}{2}\left(\frac{4 \pi \rho}{3 M}\right)^{1 / 3} \sqrt{\frac{G}{\rho}}
$$

where $\dot{\xi}$ is the reciprocal of the time required for a shear wave to traverse an atom (units are s${ }^{-1}$ ), $\rho$ is the density, and $M$ is the atomic mass (mass of a single atom). The third building block is the sub-model for $\widehat{\tau}_{s}$, given as:

$$
\widehat{\tau}_{s}=\max \left\{s_{0}-\left(s_{0}-s_{\infty}\right) \operatorname{erf}\left[\kappa \widehat{T} \ln \left(\frac{\gamma \dot{\xi}}{\dot{\epsilon}}\right)\right], s_{0}\left(\frac{\dot{\epsilon}}{\gamma \dot{\xi}}\right)^{\beta}\right\}
$$

where $\widehat{\tau}_{s}$ is a dimensionless saturation stress, and $s_{0}, s_{\infty}, \kappa, \gamma$, and $\beta$ are dimensionless parameters. The first segment of Equation (16) $\left(s_{0}-\left(s_{0}-s_{\infty}\right) \operatorname{erf}\left[\kappa \widehat{T} \ln \left(\frac{\gamma \dot{\xi}}{\dot{\epsilon}}\right)\right]\right)$ relates to plastic flow resultant from thermally activated dislocation glide. The second segment of Equation $(16)\left(s_{0}\left(\frac{\dot{\varepsilon}}{\gamma \tilde{\xi}}\right)^{\beta}\right)$ relates to plastic flow occurring in an overdriven shock regime where dislocation drag is the pre-eminate mechanism for plastic flow. The fourth building block is the sub-model for $\widehat{\tau}_{y}$, given as:

$$
\widehat{\tau}_{y}=\max \left\{y_{0}-\left(y_{0}-y_{\infty}\right) \operatorname{erf}\left[\kappa \widehat{T} \ln \left(\frac{\gamma \dot{\xi}}{\dot{\epsilon}}\right)\right], \min \left[y_{1}\left(\frac{\dot{\epsilon}}{\gamma \dot{\xi}}\right)^{y_{2}} s_{0}\left(\frac{\dot{\epsilon}}{\gamma \dot{\xi}}\right)^{\beta}\right]\right\}
$$

where $\widehat{\tau}_{y}$ is a dimensionless dynamic yield stress, and $y_{0}$, and $y_{\infty}$ are dimensionless parameters. The first segment of Equation (17) $\left(y_{0}-\left(y_{0}-y_{\infty}\right) \operatorname{erf}\left[\kappa \widehat{T} \ln \left(\frac{\gamma \dot{\xi}}{\dot{\epsilon}}\right)\right]\right)$ relates to plastic flow resultant 
from thermally activated dislocation glide. The third segment of Equation (17) $\left(s_{0}\left(\frac{\dot{\epsilon}}{\gamma \tilde{\xi}}\right)^{\beta}\right)$ relates to plastic flow occurring in an overdriven shock regime where dislocation drag is the pre-eminate mechanism for plastic flow. The second segment of Equation (17) $\left(y_{1}\left(\frac{\dot{e}}{\gamma \tilde{\xi}}\right)^{y_{2}}\right)$ allows for the potentiality of a significant upturn toward the end of the loading regime that is dominated by thermally activated dislocation glide (such as that depicted in Figure 10 of Reference [8], where $\dot{\epsilon} \sim 10^{4}$ ).

With the four building blocks now in hand, we can now proceed with a statement of the dimensionless flow stress, $\widehat{\tau}$ :

$$
\widehat{\tau}= \begin{cases}\widehat{\tau}_{s} & \text { if } a \\ \widehat{\tau}_{s}-\left(\widehat{\tau}_{s}-\widehat{\tau}_{y}\right) \exp \left(-\frac{1}{k} \epsilon\right) & \text { if } b \\ \widehat{\tau}_{s}+\frac{1}{p}\left(s_{0}-\widehat{\tau}_{y}\right) \ln \left[1-\left[1-\exp \left(-p \frac{\widehat{\tau}_{s}-\widehat{\tau}_{y}}{s_{0}-\widehat{\tau}_{y}}\right)\right] \exp \left\{-\frac{p \theta \epsilon}{\left(s_{0}-\widehat{\tau}_{y}\right)\left[\exp \left(p \frac{\widehat{\tau}_{s}-\widehat{\tau}_{y}}{s_{0}-\widehat{\tau}_{y}}\right)-1\right]}\right\}\right] & \text { if } c\end{cases}
$$

where,

a: if $\widehat{\tau}_{s}=\widehat{\tau}_{y}$;

b: if $p=0$ (Voce equation applies);

c: otherwise;

$k=\left(\widehat{\tau}_{s}-\widehat{\tau}_{y}\right) / \theta \quad(\theta$ and $p$ are dimensionless parameters).

The flow stress and saturation stress are now given as:

$$
\begin{aligned}
\sigma & =2 \widehat{\tau} G \\
\sigma_{s} & =2 \widehat{\tau}_{s} G
\end{aligned}
$$

In summary, the PTW model is a simple history-independent model that is applicable to a very wide range of strain rates. It provides both flow stress and saturation stress. It has 15 input parameters: $G_{0}, M, p, s_{0}, s_{\infty}, T_{m}, y_{0}, y_{1}, y_{2}, y_{\infty}, \alpha, \beta, \gamma, \kappa$, and $\theta$.

\subsection{MTS}

The MTS model attempts to take history into account. It is phenomenological yet is based on dislocation mechanics in the sense that it attempts to macroscopically model contributions to the flow stress from dislocation interactions with barriers, such as other disloctions, interstitial, and solute atoms. Each of these interaction contributions is quantified through an internal state variable $\widehat{\sigma}_{i}(i$ denotes the interaction number). Each of these internal state variables has its own evolution law. The internal state variables, which take into account structure evolution are coupled with constant-structure terms, $S_{i}$, $(i$ denotes the interaction number) that are primarily functions of strain rate and temperature. The model includes a term, $\widehat{\sigma}_{a}$, called the athermal threshold stress which represents dislocation interactions with long range barriers such as grain boundaries. This term is usually assumed to be constant. Independent variables are: strain, strain rate, and temperature; therefore, generically, $\sigma=\sigma(\epsilon, \dot{\epsilon}, T)$. The model is given as follows:

$$
\begin{array}{r}
G=b_{1}-\left(\frac{b_{2}}{e^{\frac{b_{3}}{T}}-1}\right) \\
\widehat{\sigma}_{s}=\widehat{\sigma}_{s 0}\left(\frac{\dot{\epsilon}^{p}}{\dot{\epsilon}_{s 0}}\right)^{\frac{k T}{G A b^{3}}} \\
X_{i}=\frac{\widehat{\sigma}_{i}}{\widehat{\sigma}_{s}} \quad \quad F\left(X_{i}\right)=\frac{\tanh \left(\alpha X_{i}\right)}{\tanh \alpha} \\
\theta_{0}=a_{0}+a_{1} \ln \dot{\epsilon}+a_{2} \sqrt{\dot{\epsilon}}
\end{array}
$$




$$
\begin{aligned}
& \frac{\partial \widehat{\sigma}_{i}}{\partial \epsilon}=\theta_{0}\left[1-F\left(X_{i}\right)\right] \widehat{\sigma}_{i}(n+1)=\widehat{\sigma}_{i}(n)+\Delta \epsilon^{p} \theta_{0}\left[1-F\left(X_{i}\right)\right] \\
& S_{i}=\left[1-\left(\frac{k T \ln \frac{\dot{\epsilon}_{i}}{\epsilon^{p}}}{G b^{3} g_{i}}\right)^{\frac{1}{q_{i}}}\right]^{\frac{1}{p_{i}}} \\
& \sigma=\widehat{\sigma}_{a}+\left(\frac{G}{G_{0}}\right) \sum_{i=1}^{N} \widehat{\sigma}_{i} S_{i}
\end{aligned}
$$

where $b_{1}, b_{2}$, and $b_{3}$ are constants in the model for the shear modulus, $G, a_{0}, a_{1}$, and $a_{2}$ are constants in the hardening function $\theta_{0}, \widehat{\sigma}_{s 0}$ is the saturation stress at $0 \mathrm{~K}, \dot{\epsilon}_{s 0}$ is the saturation stress reference equivalent plastic strain rate, $A$ is a saturation stress fitting constant, $k$ is Boltzmann's constant, $b$ is Burger's vector, $g_{i}$ is the normalized activation energy for interaction $i$, $\dot{\epsilon}_{i}$ is a thermal activation constant for interaction $i, q_{i}$ and $p_{i}$ are free energy exponents for interaction $i$, and $\alpha$ is a parameter normally set to 2 . This model requires fourteen plus $5 \mathrm{~N}$ input parameters ( $\mathrm{N}$ being the number of relevant interactions): $N, \widehat{\sigma}_{a}, \dot{\epsilon}_{i}, g_{i}, p_{i}, q_{i}, \widehat{\sigma}_{i_{0}}$ (the value of $\widehat{\sigma}_{i}$ at time $\left.t=0\right), b_{1}, b_{2}, b_{3}, \sigma_{s 0}, \dot{\epsilon}_{s 0}, A, b, a_{0}$, $a_{1}, a_{2}, \alpha$, and $G_{0}$ (the value of $G$ at $0 \mathrm{~K}$ and ambient pressure). Note that for most materials only one of the internal state variables $\left(\widehat{\sigma}_{i}\right)$ actually evolves with time (that related to dislocation-dislocation interaction), the others typically remain fixed a the given initial value. The MTS model is generally considered to be a viable option for strain rates up to about $10^{7} \mathrm{~s}^{-1}$.

\subsection{SCG}

The SCG model, like the Johnson-Cook and Preston-Tonks-Wallace models, is a simple history independent model. It includes a method for adjusting the shear modulus as well as the flow stress and includes a melt criterion but has no explicit strain rate dependence. Unlike the models previously discussed, it has explicit pressure dependence: $\sigma=\sigma(\epsilon, p, T)$. Since the model has no explicit strain rate dependence, it is necessarily limited to some degree in this regard. Its developers claim the model should be reasonably accurate so long as the strain rate is greater than about $10^{5} \mathrm{~s}^{-1}$. Steinberg and Lund [12] added a strain rate modification to the model that extends the strain rate applicability, now from $10^{4}$ to $10^{6} \mathrm{~s}^{-1}$. Our experience suggests that the model, if properly callibrated, can work reasonably well for strain rates from about $10^{3}$ to $10^{6} \mathrm{~s}^{-1}$. The basic model (that of the authors in [6]) is given as:

$$
\begin{gathered}
G=G_{0}\left\{1+A \frac{p}{\eta^{1 / 3}}-B\left(T-T_{r}\right)\right\} \\
\sigma=\sigma_{0}\left\{1+\beta\left(\epsilon^{p}+\epsilon_{i}\right)\right\}^{n}\left\{1+A \frac{p}{\eta^{1 / 3}}-B\left(T-T_{r}\right)\right\} \\
A=\frac{1}{G_{0}} \frac{\partial G}{d p} \quad B=-\frac{1}{G_{0}} \frac{\partial G}{d T} \quad \sigma_{0}\left\{1+\beta\left(\epsilon^{p}+\epsilon_{i}\right)\right\}^{n} \leq \sigma_{\max } \\
T_{m}=T_{m 0} \exp \{2 a(1-1 / \eta)\} \eta^{2\left(\Gamma_{0}-a-1 / 3\right)}
\end{gathered}
$$

where $p$ is the pressure, $T$ is the temperature, $G_{0}$ is the shear modulus at the reference state $\left(T_{r}=300 \mathrm{~K}\right.$, $\left.p=0, \epsilon^{p}=0\right), \eta$ is the compression $\left(\rho / \rho_{0}\right), \sigma_{0}$ is the reference state uniaxial yield strength, $\epsilon_{i}$ is the initial equivalent plastic strain, $n$ and $\beta$ are work hardening parameters, $\sigma_{\max }$ is the maximum value of uniaxial yield (at $T=300 \mathrm{~K}$ and $p=0$ ) that can be found in the literature, $T_{m 0}$ is the melt temperature (at $\rho=\rho_{0}$ ), $a$ is the coefficient of first-order volume correction to Grüneisen gamma, and $\Gamma_{0}$ is the reference state value of Grüneisen gamma. This model requires twelve input parameters: $G_{0}, \sigma_{0}, A, B$, $\beta, \epsilon_{i}, \sigma_{\max }, n, a, \Gamma_{0}, T_{m 0}$, and $\rho_{0}$. 


\section{PTW Model for Cerium}

As stated earlier, Plohr, Burakovsky, and Sjue [9] recently developed a callibration of the PTW model specifically for gamma phase (fcc) cerium. Within this work they treat $G_{0}$ and $T_{m}$ not as simple constants but as functions of density: $G_{0}=G_{0}(\rho)$, and $T_{m}=T_{m}(\rho)$. Following the methodology presented in Burakovsky, Greeff, and Preston, [13], Plohr et al. initiate the development of equations for $G_{0}(\rho)$ and $T_{m}(\rho)$ with the following definitions:

$$
\begin{gathered}
\frac{d \ln G_{0}(\rho)}{d \ln \rho} \equiv 2 \gamma_{G}(\rho)+\frac{1}{3} \\
\frac{d \ln T_{m}(\rho)}{d \ln \rho} \equiv 2 \gamma_{T_{m}}(\rho)-\frac{2}{3}
\end{gathered}
$$

where $\gamma_{G}(\rho)$ and $\gamma_{T_{m}}(\rho)$ are the Grüneisen gammas corresponding to the shear modulus and melt, respectively. These two Grüneisen gammas can be calculated as follows:

$$
\begin{aligned}
& \gamma_{G}(\rho)=\frac{1}{2}+\frac{\gamma_{1}}{\rho^{1 / 3}}+\frac{\gamma_{2}}{\rho^{q_{2}}} \\
& \gamma_{T_{m}}(\rho)=\frac{1}{2}+\frac{\gamma_{1}}{\rho^{1 / 3}}+\frac{\gamma_{3}}{\rho^{q_{3}}}
\end{aligned}
$$

where $\gamma_{1}=2.62215, \gamma_{2}=-5.0$, and $\gamma_{3}=-6400$. The interested reader is directed to [13] for a discussion of the methodology used, in general, to determine $\gamma_{1}, \gamma_{2}$, and $\gamma_{3}$. Solving Equations (32) and (33) yields the following for $G_{0}(\rho)$ and $T_{m}(\rho)$ :

$$
\begin{aligned}
& G_{0}(\rho)=G_{0}\left(\rho_{0}\right)\left(\frac{\rho}{\rho_{0}}\right)^{4 / 3} \exp \left\{6 \gamma_{1}\left(\frac{1}{\rho_{0}^{1 / 3}}-\frac{1}{\rho^{1 / 3}}\right)+\frac{2 \gamma_{2}}{q_{2}}\left(\frac{1}{\rho_{0}^{q_{2}}}-\frac{1}{\rho^{q_{2}}}\right)\right\} \\
& T_{m}(\rho)=T_{m}\left(\rho_{m}\right)\left(\frac{\rho}{\rho_{m}}\right)^{1 / 3} \exp \left\{6 \gamma_{1}\left(\frac{1}{\rho_{m}^{1 / 3}}-\frac{1}{\rho^{1 / 3}}\right)+\frac{2 \gamma_{3}}{q_{3}}\left(\frac{1}{\rho_{m}^{q_{3}}}-\frac{1}{\rho^{q_{3}}}\right)\right\}
\end{aligned}
$$

where the values of $\rho_{0}$ and $\rho_{m}$ were taken from the open literature, and have values of 6.775 and $6.640 \mathrm{~g} / \mathrm{cm}^{3}$, respectively. The value of $\rho_{m}$, for example, was taken from Zinov'ev [14] (pp. 191-192). At the melting point $\rho_{m}=6.640$ on the solid side and 6.687 on the liquid side, hence liquid is denser than the solid, which implies a negative slope on the melting curve. For the analytic models of [9], it is the value on the solid side that is required (i.e., $\left.6.640 \mathrm{~g} / \mathrm{cm}^{3}\right)$. The values of $G_{0}\left(\rho_{0}\right)$ and $T_{m}\left(\rho_{m}\right)$, used in [9], were $11.0 \mathrm{GPa}$ and $1071 \mathrm{~K}$, respectively.

Reasonable fits of the PTW model that bracket the experimental data available to the authors are given in Tables 1 and 2. Note that parameter set 1 matches parameter set 1 of [9], whereas parameter set 7 differs from parameter set 2 of [9]. Using parameter sets 1 and 7, comparisons are made to the empirical data in Figure 1 for $T=333 \mathrm{~K}, \rho=6.6877 \mathrm{~g} / \mathrm{cm}^{3}$, and $\dot{\epsilon}=4.2 \times 10^{3}$. The empirical data were provided to the authors by Alexander Petrovtsev [15]. Two parameter sets have been developed since the empirical data are limited and there is uncertainty associated with any extrapolation beyond the data. 
Table 1. PTW parameter set 1 .

\begin{tabular}{cc}
\hline Parameter & Value \\
\hline$p$ & 0.286 \\
$\theta$ & 0.0544 \\
$\kappa$ & 0.416 \\
$\gamma$ & 0.00271 \\
$s_{0}$ & 0.068856 \\
$s_{\infty}$ & 0.007147 \\
$y_{0}$ & 0.0685 \\
$y_{\infty}$ & 0.000746 \\
$y_{1}$ & 0.1 \\
$y_{2}$ & 1.0 \\
$\beta$ & 0.27 \\
$\alpha$ & 0.36 \\
\hline
\end{tabular}

Table 2. PTW parameter set 7.

\begin{tabular}{cc}
\hline Parameter & Value \\
\hline$p$ & 0.286 \\
$\theta$ & 0.046 \\
$\kappa$ & 0.416 \\
$\gamma$ & 0.00271 \\
$s_{0}$ & 0.096398 \\
$s_{\infty}$ & 0.01006 \\
$y_{0}$ & 0.01 \\
$y_{\infty}$ & 0.001044 \\
$y_{1}$ & 0.1 \\
$y_{2}$ & 1.0 \\
$\beta$ & 0.27 \\
$\alpha$ & 0.36 \\
\hline
\end{tabular}

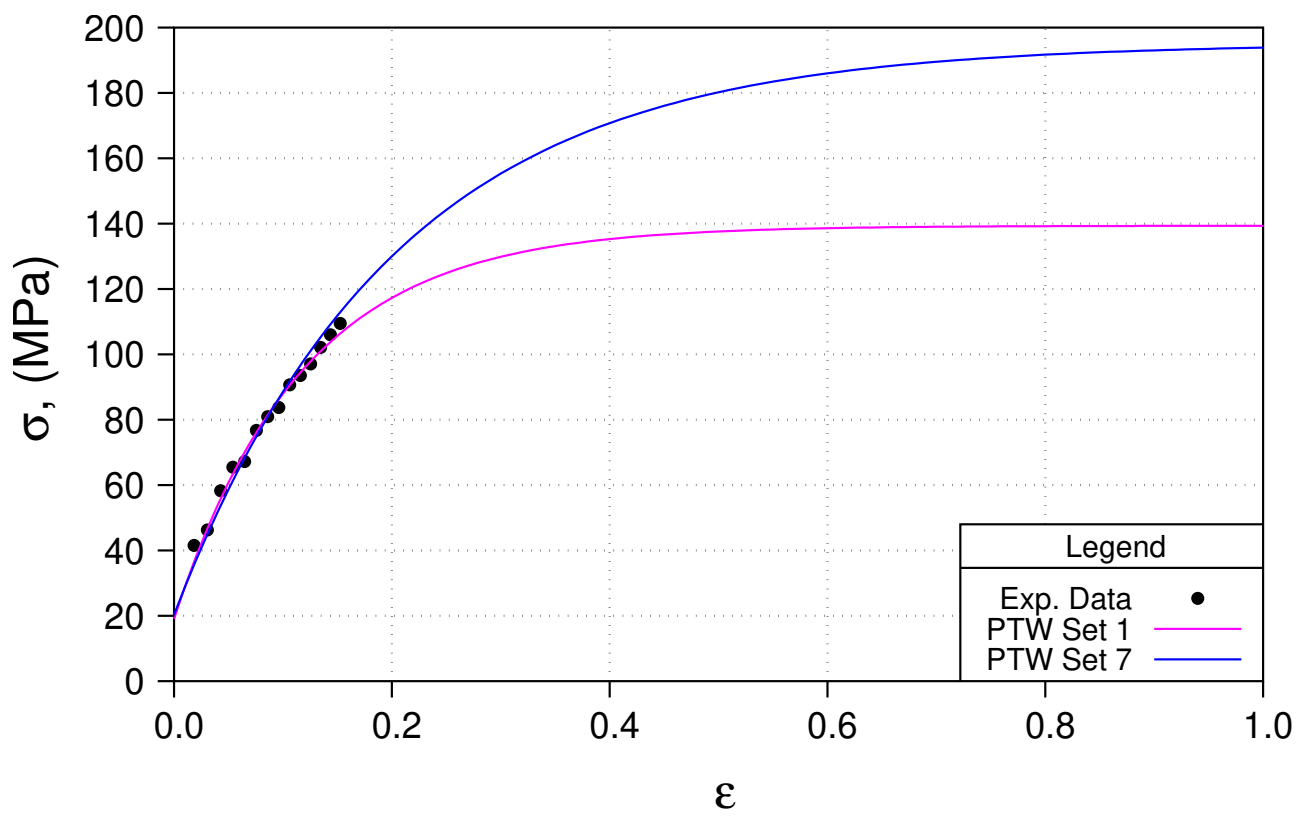

Figure 1. Approximation of Figure 3 in [9] $\left(T=333 \mathrm{~K}, \rho=6.6877 \mathrm{~g} / \mathrm{cm}^{3}, \dot{\epsilon}=4.2 \times 10^{3}\right)$. 


\section{SCG Model for Cerium}

\subsection{Melt}

Our first step in the development of an SCG model for cerium shall be directed toward melt. A determination of melt is of critical importance within the context of classical plasticity, for whenever the material is in a liquid state, the flow stress, $\sigma$, should be set to zero. Any number of melt models could be used for a determination of melt; however, in keeping with the framework established by Steinberg et al. [16], we have chosen to employ the model of Lindemann [11], which gives $T_{m}$ as a function of density:

$$
T_{m}=\frac{T_{m 0} \exp \left[2 a\left(1-\frac{1}{\eta}\right)\right]}{\left(\frac{1}{\eta}\right)^{2\left(\gamma_{0}-a-1 / 3\right)}}
$$

where

$$
\eta=\frac{\rho}{\rho_{0}}
$$

and $\rho_{0}, T_{m 0}, a$, and $\gamma_{0}$ are parameters. We shall set $\rho_{0}=6.7605 \mathrm{~g} / \mathrm{cm}^{3}$, which is the value of density at $300 \mathrm{~K}$ and $1.01325 \times 10^{-4} \mathrm{GPa}$ according to SESAME 90601 . Whereas the Grüneisen gamma used by Plohr et al. [9] is a function of density, $\gamma_{0}$ in the standard Lindemann model is simply a constant. In order to maintain some consistency with the work in [9], we shall base our value of $\gamma_{0}$ upon Equation (35), using our value of $\rho_{0}$, that is $6.7605 \mathrm{~g} / \mathrm{cm}^{3}$, and shall set the values of $\gamma_{1}, \gamma_{3}$, and $q_{3}$ to the values used in [9], which have been provided for the convenience of the reader in Section 3. By this calculation, the value of $\gamma_{0}$ is -0.203881 . We shall set the value of $T_{m 0}$ to $1000 \mathrm{~K}$. The value of $a$, is then determined by fitting the model to the data. A value of $a=-3.60$ fits the data reasonably well. Thus the Lindemann parameter set is: $\rho_{0}=6.7605 \mathrm{~g} / \mathrm{cm}^{3}, T_{m 0}=1000 \mathrm{~K}, a=-3.60$, and $\gamma_{0}=-0.203881$. Using this parameter set, a plot of melt versus density has been generated and is presented in Figure 2, along with a plot of melt versus density, generated using Equation (37) with a few points calculated using quantum molecular dynamics (QMD) and some experimental data.

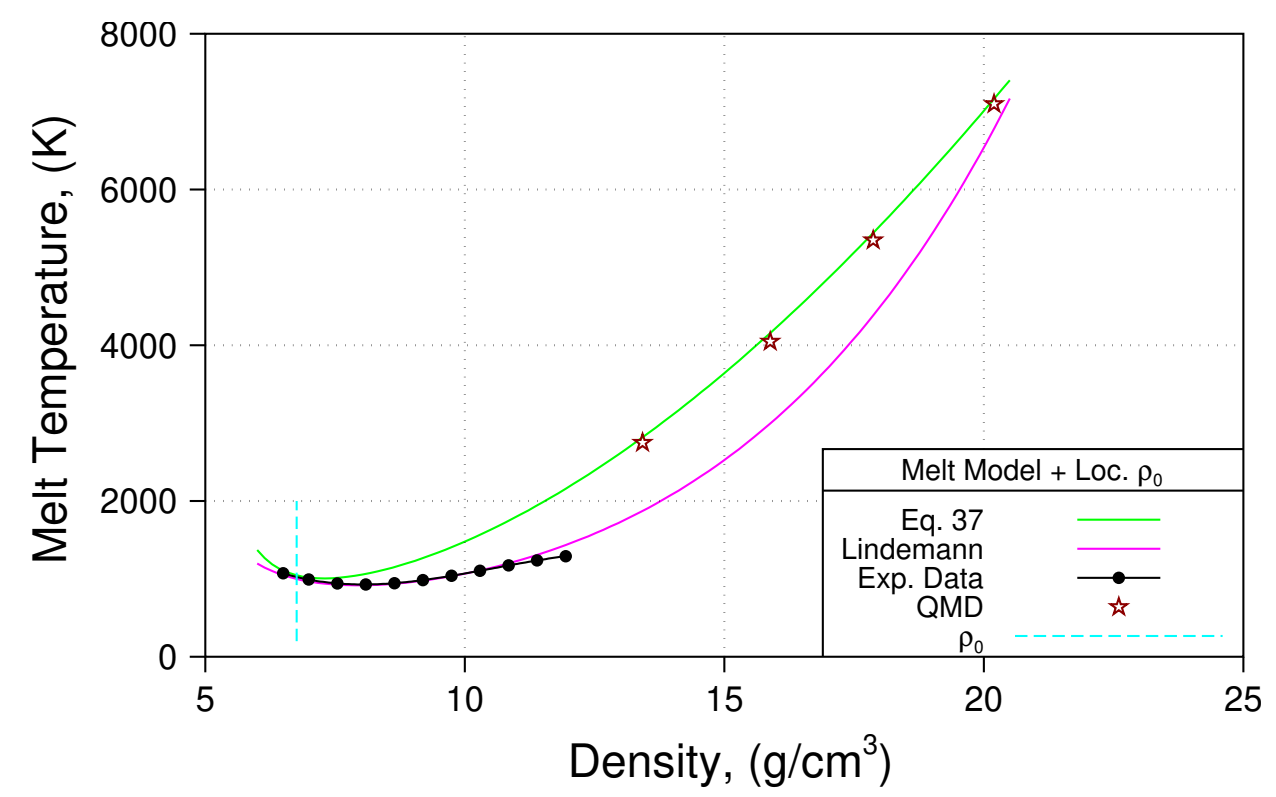

Figure 2. Melt temperature as a function of density (Lindemann parameter set 1).

Note that the Lindemann fit depicted in Figure 2 matches the experimental data reasonably well. It also extrapolates to the fit of Equation (37) and the QMD calculations at very high densities. It does not, however, for the most part, match the fit of Equation (37). Note also that, while the fit of 
Equation (37) matches the QMD calculations exceedingly well, it does not match the experimental data nearly so well as does the Lindemann fit. The third author recognized this and identified the source of this deficiency as resultant from not carrying a sufficient number of terms in the Grüneisen gamma. The three-term representation of the Grüneisen gamma (Equation (35)) is cast in terms of a Taylor-Maclaurin power series [17]:

$$
\gamma_{T_{m}}(\rho)=0.5+\sum_{i=1}^{\infty} \frac{\gamma_{i}}{\rho^{i / 3}}
$$

If instead of truncating the series at three, we truncate at five, and obtain a much better prediction of melt, one that matches the experimental data and also extrapolates to the QMD calculations reasonably well. Truncating at five we have:

$$
\gamma_{T_{m}}(\rho)=0.5+\frac{\gamma_{1}}{\rho^{1 / 3}}+\frac{\gamma_{2}}{\rho^{2 / 3}}+\frac{\gamma_{3}}{\rho}+\frac{\gamma_{4}}{\rho^{4 / 3}}
$$

and

$$
T_{m}(\rho)=T_{m}\left(\rho_{m}\right)\left(\frac{\rho}{\rho_{0}}\right)^{1 / 3} \exp \left\{\begin{array}{c}
6 \gamma_{1}\left(\frac{1}{\rho_{0}^{1 / 3}}-\frac{1}{\rho^{1 / 3}}\right) \\
-3 \gamma_{2}\left(\frac{1}{\rho_{0}^{2 / 3}}-\frac{1}{\rho^{2 / 3}}\right) \\
+2 \gamma_{3}\left(\frac{1}{\rho_{0}}-\frac{1}{\rho}\right) \\
-3 / 2 \gamma_{4}\left(\frac{1}{\rho_{0}^{4 / 3}}-\frac{1}{\rho^{4 / 3}}\right)
\end{array}\right\}
$$

where $\rho_{0}=6.64 \mathrm{~g} / \mathrm{cm}^{3}, T_{m}\left(\rho_{m}\right)=1071 \mathrm{~K}, \gamma_{1}=2.62215, \gamma_{2}=28.4194, \gamma_{3}=200.641$, and $\gamma_{4}=311.183$.

In Figure 3, melt (as predicted using Equation (42)), is added to what was presented in Figure 2, and indeed, it is shown that this curve matches the data well and extrapolates to the QMD calculations in reasonable fashion.

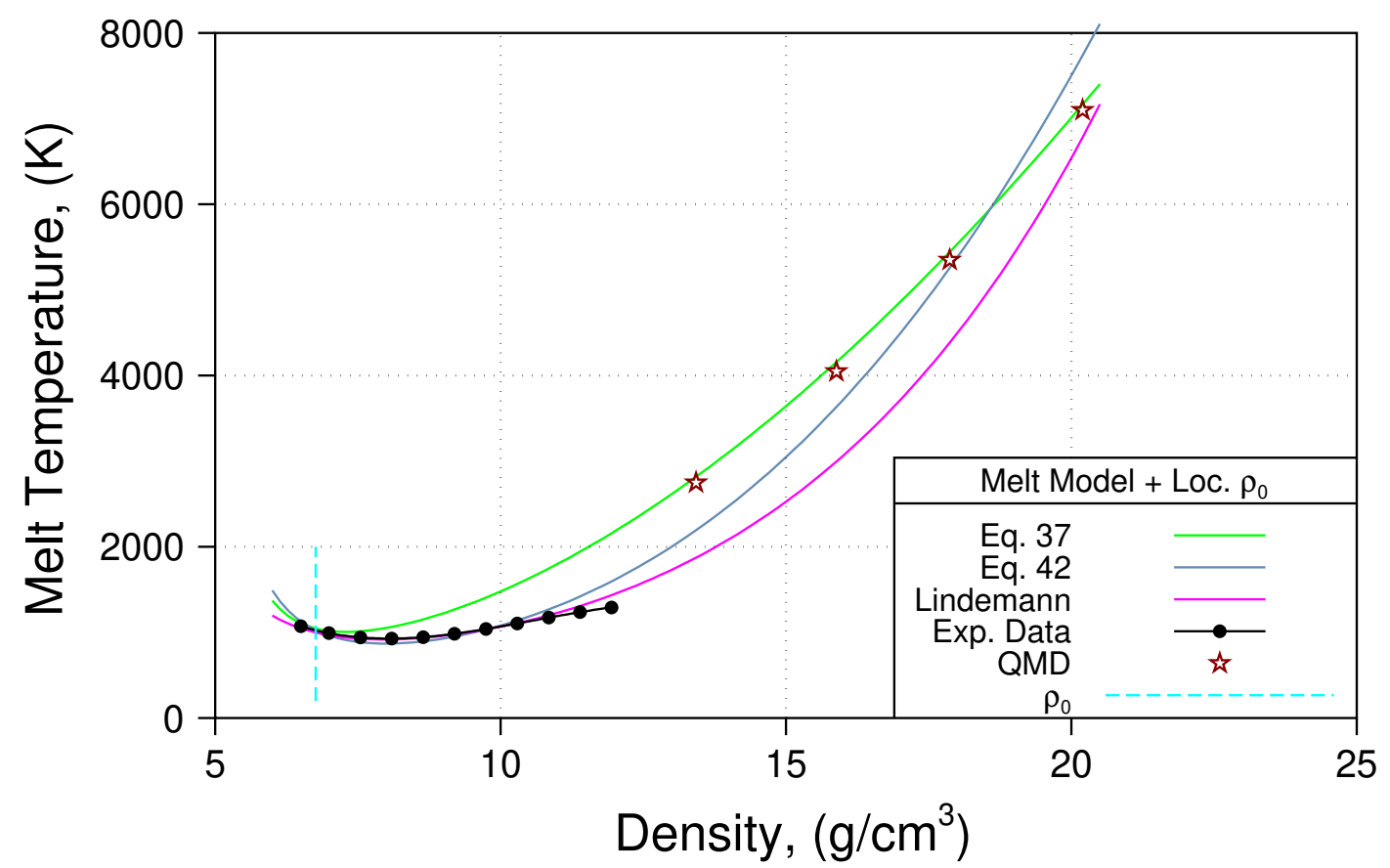

Figure 3. Melt calculated using 5-term Grüneisen added to what was presented in Figure 2. 


\subsection{Shear Modulus}

Our second step in the development of a SCG model for cerium is directed toward the shear modulus. Here, basing our model on that of [9] is a bit convoluted since, in [9], $G=G(\rho, T)$, whereas under the SCG framework $G=G(\rho, p, T)$ and pressure plays a prominant role in the model (see Equation (28)). Plohr et al. expressed $G=G(\rho, T)$ as:

$$
G(\rho, T)=G_{0}(\rho)\left(1-\alpha \frac{T}{T_{m}(\rho)}\right)
$$

For the SCG model of shear modulus, we require five parameters (see Equation (28)): $A, B, G_{0}$, $\rho_{0}$, and $T_{r}$ ). We shall set $\rho_{0}=6.7605 \mathrm{~g} / \mathrm{cm}^{3}$ as in the previous section, and set $T_{r}$ to $300 \mathrm{~K}$. We shall set $G_{0}$ to the value calculated using Equation (36), which turns out to be $10.94 \mathrm{GPa}$. Note that, in the calculation of $G_{0}$, we set the values of $G_{0}\left(\rho_{0}\right), \rho_{0}, \gamma_{1}, \gamma_{2}$, and $q_{2}$ to the values used in [9]; namely, 11.0 GPa, $6.775 \mathrm{~g} / \mathrm{cm}^{3}, 2.62215,-5.0$, and 1.0, respectively. Parameter $B$ can be calculated directly from Equation (43) as follows:

$$
\begin{aligned}
B & =-\frac{1}{G_{0}(\rho)} \frac{\partial G}{\partial T} \\
& =\left(-\frac{1}{G_{0}(\rho)}\right)\left(-\frac{G_{0}(\rho) \alpha}{T_{M}(\rho)}\right) \\
& =\frac{\alpha}{T_{m}(\rho)} \\
B & =3.435 \times 10^{-4} \mathrm{~K}^{-1}
\end{aligned}
$$

The determination of parameter $A$ is somewhat less straightforward. Recall that $A=1 / G_{0} \partial G / \partial p$ (see Equation (30)). Since $G$ in [9] is not a function of pressure, we are unable to calculate its value directly as was done above for parameter $B$. We need $G=G(\rho, p, T)$ from which we can determine $\partial G / \partial p$. To accomplish this, we set $T=300 \mathrm{~K}$ and we use SESAME 90601 to obtain $p=p(\rho)$. Then for any value of $\rho$ we know the corresponding value of $p$. Using this information and Equation (36) we can construct $G=G(p)$ at $\rho=\rho_{0}$ and $T=300 \mathrm{~K}$. We can then fit a straight line to that construct, from which $\partial G / \partial p$ is easily determined. That construct so determined along with a linear fit to that construct is shown in Figure 4. The value of $A$ (i.e, the slope of the linear fit shown in Figure 4) is $0.0676322 \mathrm{GPa}^{-1}$, which we shall treat as $0.0676 \mathrm{GPa}^{-1}$ in subsequent calculations.

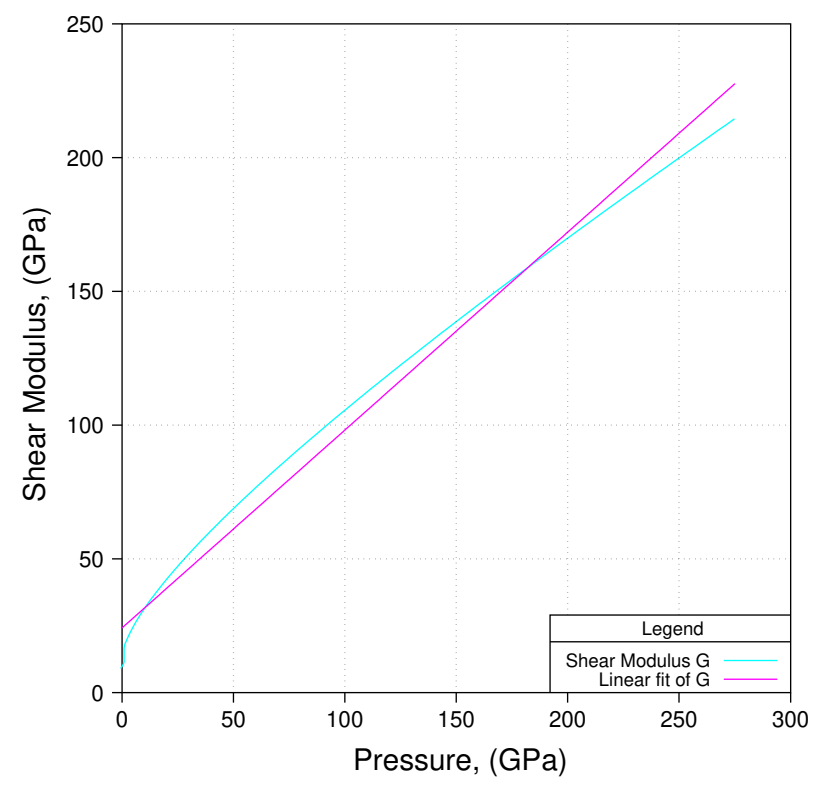

Figure 4. Shear modulus as a function of pressure. 
The SCG model of the shear modulus determined as described above is compared to the model of Plohr et al. [9] (Equation (43)) in Figure 5. The fact that $G=G(\rho)$ (for constant $T$ ) differs significantly between the two models shown in Figure 5 is not of particular concern. Recall that, in the SCG model, $G=G(\rho, p, T)$, whereas in the model of [9], $G=G(\rho, T)$. Consequently, it is not surprising that there are some differences in this regard. Note that it is quite possible that, with some other relationship between density and pressure (we chose to base that relationship on SESAME 90601), we would see a smaller difference between the curves shown in Figure 5. It is also possible that, if a five-term Taylor-Maclaurin power series had been used in the development of Equation (36) instead of a three-term series, this might also have served to result in a smaller difference between the two curves. At any rate, our objective is not to match the curve generated by Equation (43), but rather to develop a model based in large part upon SESAME 90601 that is logical and therefore justifiable. For given values of density and pressure (e.g., $6.6705 \mathrm{~g} / \mathrm{cm}^{3}$ and $1.01325 \times 10^{-4} \mathrm{GPa}$, respectively), there is very little difference between plots of $G$ versus $T$ derived from $G=G(\rho, T)$ as expressed in Equation (43) and from $G=G(\rho, p, T)$ as expressed in our SCG model, as is shown in Figure 6. Again, the difference shown is Figure 6 is of little concern. In summary, the five parameters of our SCG model of shear modulus are as follows: $A=0.0676 \mathrm{GPa}^{-1}, B=3.435 \times 10^{-4} \mathrm{~K}^{-1}, G_{0}=10.94 \mathrm{GPa}, \rho_{0}=6.7605 \mathrm{~g} / \mathrm{cm}^{3}$, and $T_{r}=300 \mathrm{~K}$.

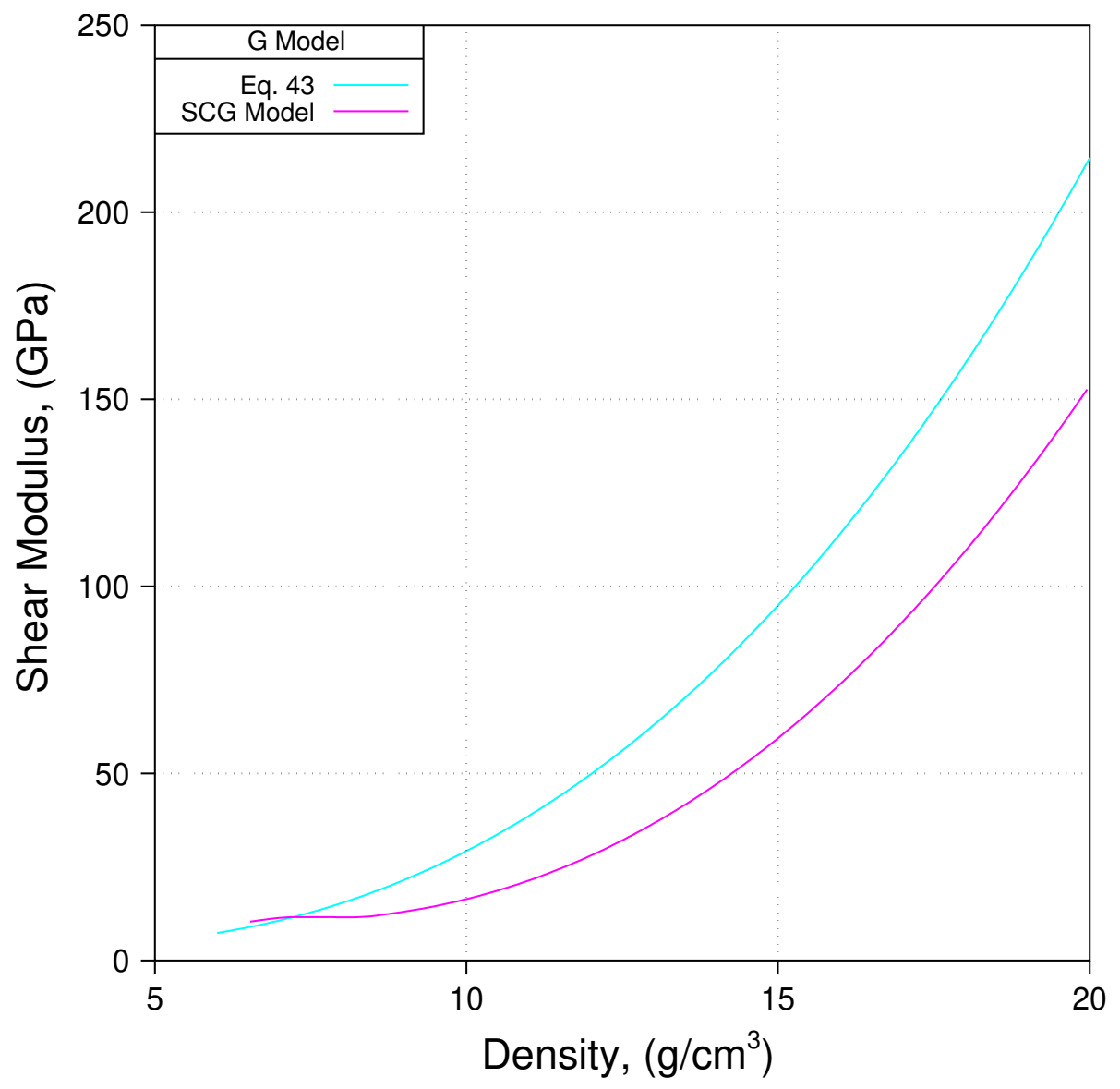

Figure 5. Shear modulus as a function of density. 


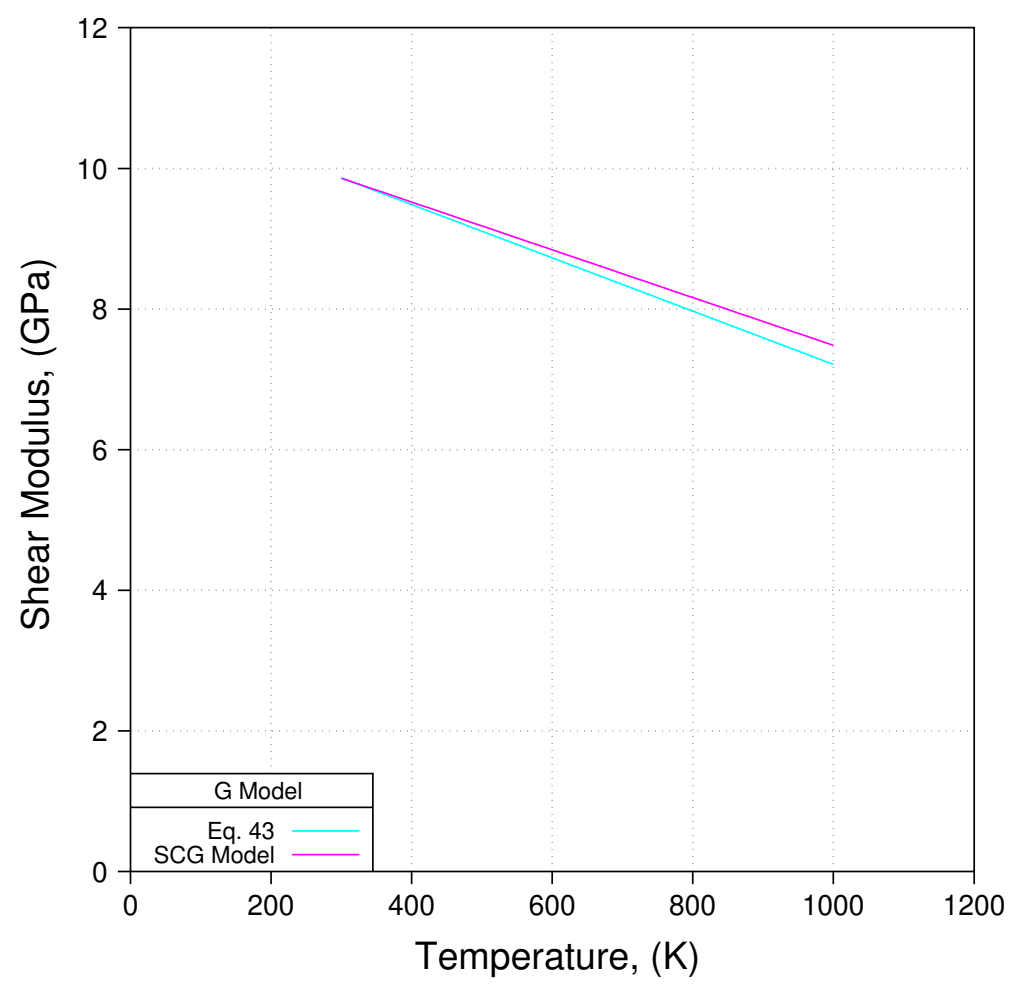

Figure 6. Shear modulus as a function of temperature.

\subsection{Flow Stress}

The third and final step in our development of a SCG model is the flow stress. For this, we must determine five additional parameters (see Equations (28)-(30)): $\sigma_{0}, \beta, \epsilon_{i}, \sigma_{\max }$, and $n$. Consistent with Figure 1 we shall set $\sigma_{0}$ to $20.36 \mathrm{MPa}$. In keeping with common convention (see Steinberg [16]), we shall set the residual (or initial strain) $\epsilon_{i}$ to 0 . This leaves only the work hardening parameters $\left(\beta, n\right.$, and $\left.\sigma_{\max }\right)$ to be determined. These parameters affect the shape of the predicted flow-stress curve. Two sets of values were determined, one to approximate the shape of the PTW Set 1 curve, shown in Figure 1, the other to approximate the shape of the PTW Set 7 curve shown in Figure 1. Parameter $\beta$ is hereby set to a value of 140 for both cases. Parameter $n$ is hereby set to a value of 0.555 to approximate the PTW Set 7 curve, and a value of 0.538 to approximate the PTW Set 1 curve. Parameter $\sigma_{\max }$ is hereby set to $196 \mathrm{MPa}$ in order to approach the PTW Set 7 curve at large strain, and to a value of 140.5 MPa in order to approach the PTW Set 1 curve at large strain.

This completes our development of a SCG model for cerium. A full set of parameters for the now complete model (actually two models: one representing a lower bound and the other an upper bound on flow stress) are given in Table 3. In the course of this work, many different parameter sets were evaluated. The set numbers given in Table 3 are merely book keeping identifiers, included for the convenience of the authors, and have no significance beyond a desire for careful documentation. Plots of both lower and upper bound models are provided in Figure 7.

\subsection{Experimental Methods}

Since the focus of this work is on model development and validation, as opposed to, for example, experimental methods, we shall forego a lengthy discussion pertaining to the experimental methods that might be employed in the validation of hardening models. Instead, we refer the reader to the work of Zocher et al. [18], wherein experimental methodologies that may be employed for this purpose are promulgated. 
Table 3. SCG model for cerium.

\begin{tabular}{ccc}
\hline Parameter & Comment & Value \\
\hline Lindemann Melt & \\
$\rho_{0}$ & $6.7605 \mathrm{~g} / \mathrm{cm}^{3}$ \\
$\gamma_{0}$ & -0.203881 \\
$T_{m 0}$ & $1000 \mathrm{~K}$ \\
$a$ & -3.60 \\
\hline Shear Modulus & \\
$A$ & & $0.0676 \mathrm{GPa}^{-1}$ \\
$B$ & & $3.435 \times 10^{-4} \mathrm{~K}^{-1}$ \\
$G_{0}$ & $10.94 \mathrm{GPa}$ \\
$\rho_{0}$ & $6.7605 \mathrm{~g} / \mathrm{cm}^{3}$ \\
$T_{r}$ & $300 \mathrm{~K}$ \\
\hline Flow Stress & & \\
$\sigma_{0}$ & & $0.02036 \mathrm{GPa}$ \\
$\beta$ & & 140 \\
$\epsilon_{i}$ & & 0 \\
$\sigma_{\max }$ & lower bound (Param. Set 158) & $0.1405 \mathrm{GPa}$ \\
$\sigma_{\max }$ & upper bound (Param. Set 155) & $0.196 \mathrm{GPA}$ \\
$n$ & lower bound (Param. Set 158) & 0.538 \\
$n$ & upper bound (Param. Set 155) & 0.555 \\
\hline
\end{tabular}

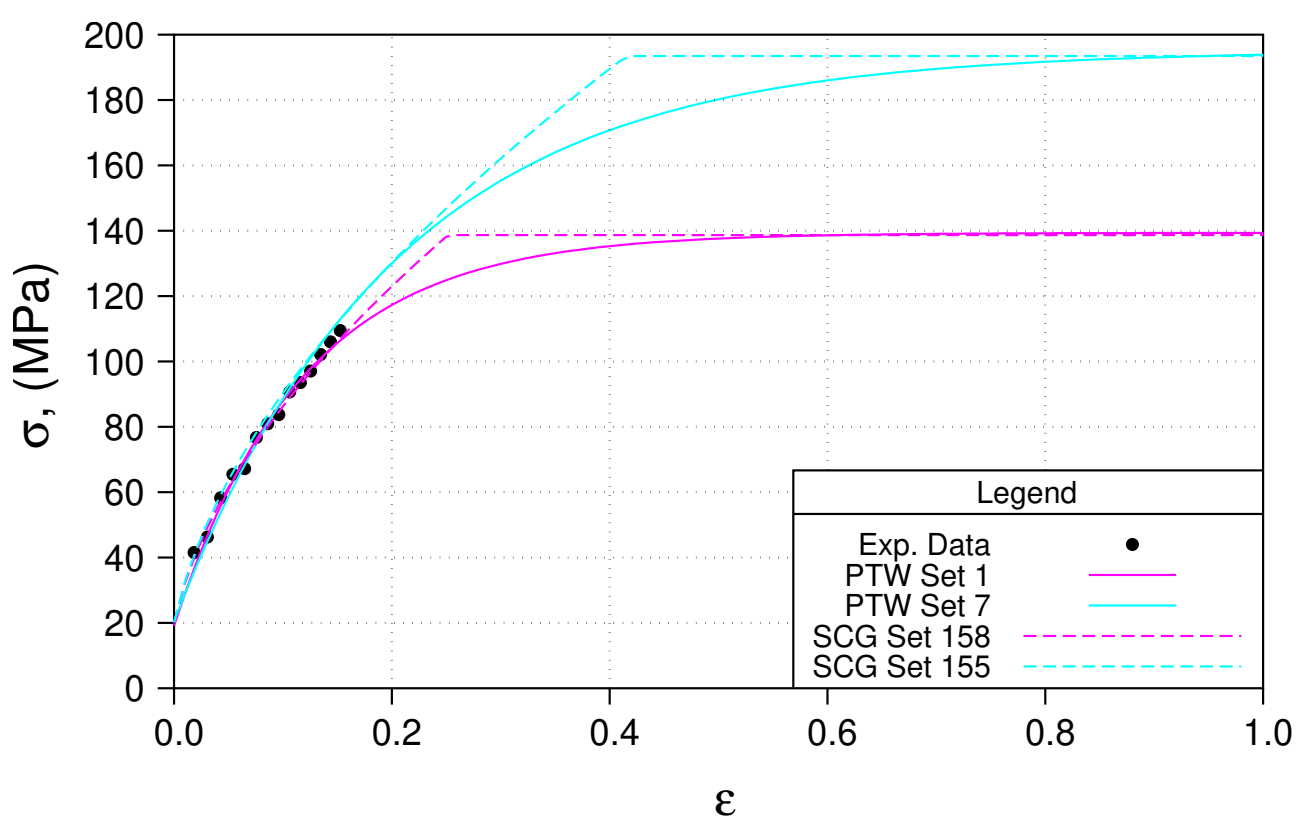

Figure 7. Flow stress as a function of strain.

\section{Conclusions}

To summarize, an SCG model for the shear modulus and flow stress that incorporates a Lindemann model for melt has been developed. Actuallyn two SCG models have been developed, one as a lower bound, one as an upper bound. These models were developed using the PTW model of Plohr et al. [9] as a starting point. Having said that, it should be stated that it was not the intent herein to match the models of [9] exactly. As was the case with the models developed in Plohr et al. [9], the current model is only strictly applicable to the relatively low pressure $\gamma$-phase of cerium and to relatively low values of equivalent strain (up to about 0.18 ). It should be emphasized that the extrapolations beyond available data (e.g., shown in Figures 1 and 7), are merely upper and lower bound guesses. Models that are validated to higher values of strain await the availability of additional empirical data. 
Author Contributions: The PTW model for cerium was developed by J.N.P. and L.B. The SCG model for cerium was developed by M.A.Z. The paper was written primarily by M.A.Z. All authors have read and agreed to the published version of the manuscript.

Funding: This work was supported by the US Department of Energy and the National Nuclear Security Administration.

Conflicts of Interest: The authors declare no conflict of interest.

\section{References}

1. Malvern, L.E. Introduction to the Mechanics of a Continuous Medium; Prentice-Hall: Englewood Cliffs, NJ, USA, 1969.

2. Lubliner, J. Plasticity Theory; Macmillan: New York, NY, USA, 1990.

3. Truesdell, C. The Elements of Continuum Mechanics; Springer: New York, NY, USA, 1966.

4. Zocher, M.A.; Maudlin, P.J.; Chen, S.R.; Flower-Maudlin, E.C. An Evaluation of Several Hardening Models using Taylor Cylinder Impact Data. In Proceedings of the ECCOMAS 2000, European Congress on Computational Methods in Applied Sciences and Engineering, Barcelona, Spain, 11-14 September 2000.

5. Johnson, G.R.; Cook, W.H. A Constitutive Model and Data for Metals Subjected to Large Strain High Strain Rates, and High Temperatures. In Proceedings of the 7th International Symposium on Ballistics, The Hague, The Netherlands, 19-21 April 1983.

6. Steinberg, D.G.; Cochran, S.G.; Guinan, M.W. A constitutive model for metals applicable at high-strain rate. J. Appl. Phys. 1980, 51, 1498-1504. [CrossRef]

7. Follansbee, P.S.; Kocks, U.F. A constitutive description of the deformation of copper based on the use of the mechanical threshold stress as an internal state variable. Acta Metall. 1988, 36, 81-93. [CrossRef]

8. Preston, D.L.; Tonks, D.L.; Wallace, D.C. Model of plastic deformation for extreme loading conditions. J. Appl. Phys. 2003, 93, 211-220. [CrossRef]

9. Plohr, J.; Burakovsky, L.; Sjue, S. The Preston-Tonks-Wallace Model Parameterization for Gamma (FCC)-Phase Cerium. In Proceedings of the Shock Compression of Condensed Matter, Portland, OR, USA, 16-21 June 2019.

10. Zocher, M.A.; Kenamond, M.A.; Hammerberg, J.E. Toward Numerical Validation of a New Multiscale Friction Model. In LANL Report No. LAUR-18-28995; Los Alamos National Laboratory: Los Alamos, NM, USA, 2018.

11. Lindemann, F. Über die Berechnung Molekularer Eigenfrequenzen (The calculation of molecular vibration frequencies). Phys. Z. 1910, 11, 609-612.

12. Steinberg, D.G.; Lund, C.M. A Constitutive Model for Strain Rates from $10^{-4}$ to $10^{6}$. J. Appl. Phys. 1989, 65, 1528-1533. [CrossRef]

13. Burakovsky, L.; Greeff, C.W.; Preston, D.L. Analytic model of the shear modulus at all temperatures and densities. Phys. Rev. B 2003, 67, 4107. [CrossRef]

14. Zinov'ev, V.E. Handbook of Thermophysical Properties of Metals at High Temperatures; Nova Science Publishers: Hauppauge, NY, USA, 1996.

15. Petrovtsev, A.V. $\sigma-\epsilon$ Diagrams of the High-Strain Rate Behavior of Cerium under Ambient and Elevated Temperatures. In VNIITF Report No. Agreement 4783-000-99-35, Task order 010, Modification 7, Deliverable 5.2.2; Zababakhin All Russia Scientific Research Institute of Technical Physics (VNIITF): Snezhinsk, Russia, 2013.

16. Steinberg, D.J. Equation of State and Strength Properties of Selected Materials. In LLNL Report No. UCRL-MA-106439, Change 1; Lawrence Livermore National Laboratory: Livermore, CA, USA, 1996.

17. Tierney, J.A. Calculus and Analytic Geometry; Allyn and Bacon: Boston, MA, USA, 1966; pp. 582-587.

18. Zocher, M.A.; Raevsky, V.A.; Ignatova, O. Deviatoric Constitutive Model: Domain of Strain Rate Validity. In Advances in Materials Science; SNL Publication SAND 2009-4908 P, Sandia National Laboratory: Albuquerque, NM, USA, 2009.

Publisher's Note: MDPI stays neutral with regard to jurisdictional claims in published maps and institutional affiliations.

(C) 2020 by the authors. Licensee MDPI, Basel, Switzerland. This article is an open access article distributed under the terms and conditions of the Creative Commons Attribution (CC BY) license (http:/ / creativecommons.org/licenses/by/4.0/). 